\title{
Conferencia Héctor Orrego Puelma 2005: Ancestros de la Neumología
}

\author{
EDGARDO CRUZ M.*
}

Professor Héctor Orrego-Puelma Lecture 2005: Ancestors of the Respiratory Disease Specialty

Por celebrarse este año 2005 los 75 años de nuestra Sociedad se me pidió abordar en la Conferencia Héctor Orrego Puelma algún tema relacionado con nuestra especialidad. Como Manuel Barros se había referido a la historia de la Sociedad en el editorial del primer número de la Revista de 2005 y la complementó en forma gráfica y amena en las Jornadas de Invierno, Gladio Mena publicó un exhaustivo estudio de la historia de la especialidad en Chile en 1989 y Victorino Farga publicó una muy bien resumida historia de la tuberculosis en 2004, para evitar repeticiones decidí indagar sobre cómo, por qué y por quienes se generó la especialidad en el mundo. Creo conveniente y justo tomar conciencia de lo que debemos a nuestros ancestros y el pasado nos puede evitar repetir errores y nos brinda modelos que nos pueden ayudar en lo que hoy queremos hacer.

En el esquema de la Figura 1 la tuberculosis se muestra como nuestra más remota raíz. Si bien esta enfermedad está presente en la historia de la humanidad desde hace, por lo menos, 5000 años, sólo en el siglo XIX aparecen médicos que se dedican exclusivamente a ella por su alta prevalencia, complejidad y rebeldía y con ello nace la Tisiología, disciplina que es moldeada a lo largo de ese siglo por una serie de im-

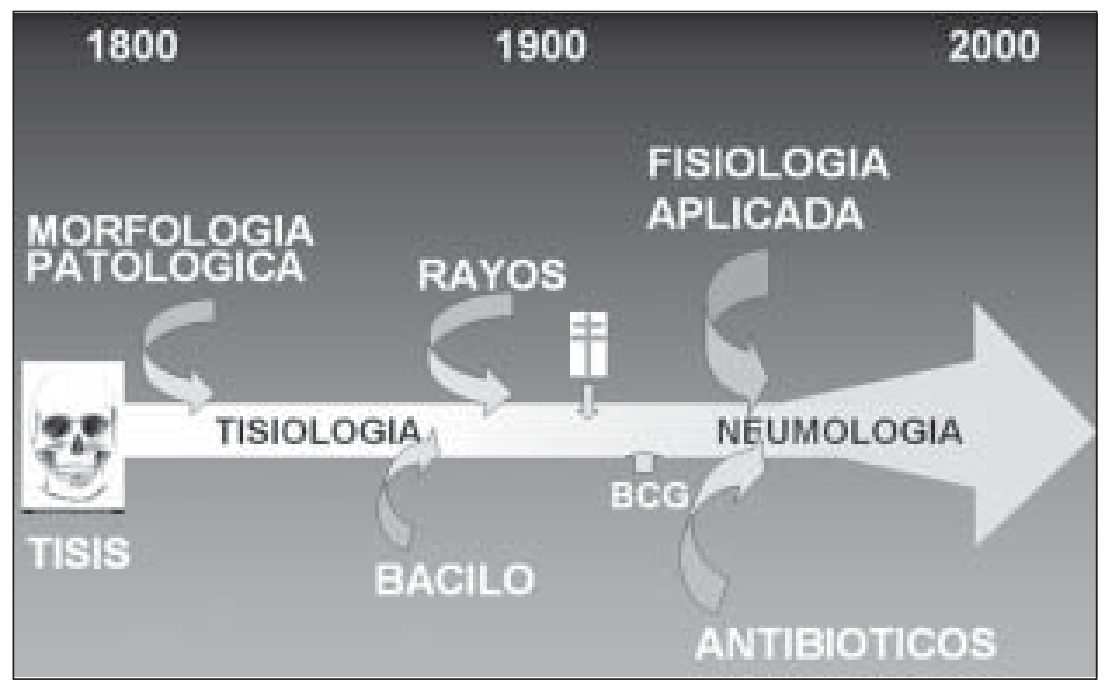

Figura 1. Tuberculosis en la Historia de la humanidad.

* Departamento de Enfermedades Respiratorias, Facultad de Medicina, Pontificia Universidad Católica de Chile.

Conferencia pronunciada en el XXXVIII Congreso Chileno de Enfermedades Respiratorias, Puyehue, Osorno, 3 de noviembre, 2005. 
pactos de efecto variable para terminar por constituir en el siglo XX una de las vertientes fundamentales de la neumología.

A principios del siglo XIX René Laennec como clínico, atendía a los tísicos y como patólogo hacía las autopsias de los muchos que morían. A través de una cuidadosa correlación anátomo-clínica, pudo dejar en claro que varias enfermedades que eran consideradas como distintas, eran sólo diferentes formas o localizaciones de la temida tisis, a la cual, unas dos décadas después, Schonlein dio su nombre actual de tuberculosis. Su doble condición de clínico y patólogo, también dio base a Laennec para establecer, con notable claridad, las bases de la auscultación pulmonar, herramienta diagnóstica básica de los médicos por largos años, a pesar de las deformaciones a que la sometieron sus traductores y contribuidores teóricos. Como precio de su dedicación murió de tuberculosis.

Aunque anteriormente se había especulado que la tuberculosis era contagiosa, fue pasada la mitad del siglo, cuando esto fue demostrado por Juan Antonio Vilemin, médico militar francés, quien provocó tuberculosis en conejos inoculando productos biológicos de tuberculosis humana. A pesar de su objetividad, la prueba fue en general rechazada, porque se contradecía con la teoría que la tisis era producto de una constitución especial.

Sin duda, el hito más importante en el conocimiento de la tuberculosis fue el descubrimiento del germen causal por Robert Koch (Figura 2). A los 42 años, con un rigor metódico extraordinario, no sólo tiñó e identificó al bacilo en su modesto laboratorio personal, continuando a Pasteur, estableció las bases conceptuales y técnicas de la bacteriología y planteó los postulados que permiten afirmar la relación causal entre un germen y determinada enfermedad. En 1882 presentó su estudio en la Sociedad Médica de Berlín, con un impacto tal que a nadie se le ocurrió aplaudir. Sobre la actitud de Virchow, autoridad mundial de la medicina en esos tiempos, hay dos versiones: una que se levantó y se retiró sin decir nada y otra que no asistió. Lo cierto es que siempre manifestó su escepticismo hablando del "así llamado bacilo de la tuberculosis". A pesar de ello, a los tres años el bacilo de Koch se había impuesto sin discusión, plazo impresionante al lado de los 150 años que demoró la circulación, descubierta por Harvey, en ser aceptada universalmente.

A pesar de estos avances la tuberculosis seguía siendo la ominosa peste blanca: a fines del siglo XIX a los 5 años de contraída una tuber- culosis, la mitad de los enfermos estaban muertos, una cuarta parte continuaban activos y el otro cuarto estaban detenidos, sin seguridad de haber curado definitivamente. Aunque el azote era mucho peor entre los pobres, la enfermedad no respetaba clases ni fama como lo ilustran algunos ejemplos (Tabla 1).

Entre los mecanismos a los cuales la sociedad, principalmente la europea entonces dominante en el mundo, recurrió para enfrentar el ataque ante el cual se encontraba inerme, cabe destacar, por curioso, el de la idealización de la enfermedad.

Por los personajes que afectó se generalizó la idea que la tuberculosis favorecía el desarrollo del genio y que el aspecto pálido y consuntivo era una forma de belleza romántica que el maquillaje de moda debía imitar. Lord Byron, el poeta, decía que deseaba morir de tuberculosis para que todas las mujeres lo recordaran como interesante. El enfermo tuberculoso pasó a ser tema de las artes: en la literatura la madre de Cosette, heroína de "Los Miserables" de Víctor Hugo y la protagonista de "La Dama de las Camelias" de Alejando Dumás mueren de esta enfermedad y en música la agonía de ésta última es inmortalizada en la opera "La Traviata" de Verdi. El pintor Edward Munch reprodujo en sus cuadros la enfermedad y muerte de su madre y hermana.

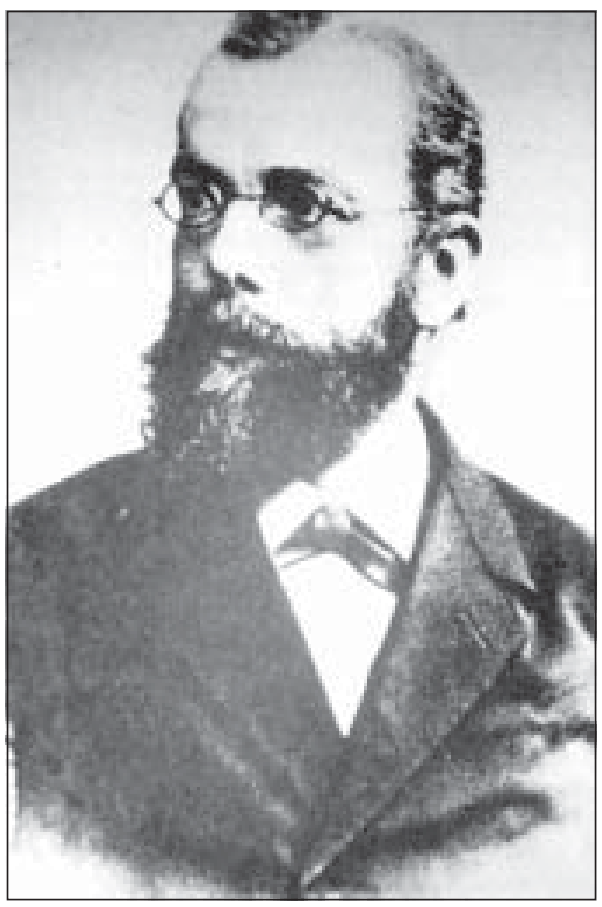

Figura 2. Robert Koch (1843-1910). 
Tabla 1. Personalidades afectadas por la tuberculosis

Enfermos de TBC
Ludwig van Beethoven
Frederic Chopin
Marie Curie
René Descartes
Fedor Dostoievski
Johann Goethe
Guy de Maupassant
Napoleón Bonaparte
Niccolo Paganini
H. van Rijn Rembrandt
Francois Voltaire
Muertos de TBC
Anton Chekov
John Keats
René Laennec
Edgar Allan Poe
Cardenal Richelieu
Johann von Schiller
Walter Scott
Santa Teresa de Lissieux
Franz Kafka
George Orwell
Vivien Leigh

Otra línea de acción más racional, aunque con un respaldo científico débil, fue el desarrollo de sanatorios. El primero fue fundado en 1859 por Herman Brehmer estudiante de botánica, cuya tuberculosis se habría curado en un viaje a los Himalayas y que luego estudió medicina. Su relativo éxito estimuló la sucesiva creación de nuevos sanatorios, principalmente en altura, que sobre la base de consideraciones teóricas, implantaron diferentes y contradictorios programas de actividad física, alimentación, exposición climática, etc.

Especial fama lograron los sanatorios de Davos en Suiza, pueblo donde el número de enfermos llegó a superar al de habitantes. Thomas Mann, tras acompañar seis meses a su mujer en uno de estos sanatorios, escribió su famosa novela "La montaña mágica" en la cual describe con sorprendente exactitud y minucia los aspectos médicos y el Dr. Sótero del Río, fundador de nuestra Sociedad, se trató en Davos donde, además de sanar, se transformó en tisiólogo.
En los últimos años el quehacer de los sanatorios se fue haciendo más técnico y en ellos se desarrollaron el neumotoráx terapéutico y los primeros pasos de la cirugía de tórax.

El segundo gran impacto que experimentó la tisiología tuvo lugar a fines del siglo XIX y se debió a Wilhelm Conrad Roentgen (Figura 3).

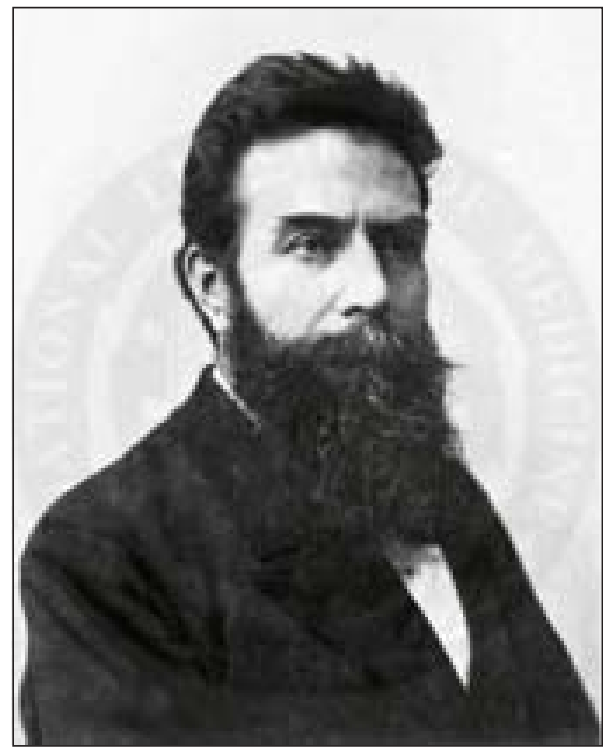

Figura 3. Wilhelm Conrad Roentgen (18451923).

Este era un hombre exageradamente reservado que desató uno de los eventos científicos y comunicacionales más grandes de la historia e inició una revolución tecnológica que aun está en plena expansión. Como físico se interesaba por los rayos catódicos, generados en los tubos de Crookes por una chispa eléctrica que saltaba desde el ánodo al cátodo a través del vacío. Los efectos de estos rayos se manifiestan hasta una distancia de 10-15 cm y son capaces de velar el papel fotográfico y producir fluorescencia en el vidrio y en pantallas especiales. El 8 de Noviembre de 1885 le llamó la atención la aparición de fluorescencia en un papel impregnado con platino-cianuro de bario que estaba a 2 metros del tubo de Crookes, demasiado lejos como para ser atribuido a rayos catódicos. Anteriormente, Crookes había observado algo similar al velarse unas placas fotográficas guardadas a distancia, pero se limitó a trasladarlas a otra parte. Como dijo Pasteur, "la suerte favorece a los más preparados".

Entusiasmado e intrigado, Roentgen se ence- 
rró, literalmente, en su laboratorio durante 40 días e hizo toda clase de experimentos para caracterizar estos rayos desconocidos que llamó rayos $\mathrm{X}$. Probó el efecto de campos magnéticos y eléctricos, prismas y la opacidad a rayos de cuanto tuvo a su alcance, incluso la mano de su esposa (Figura 4).

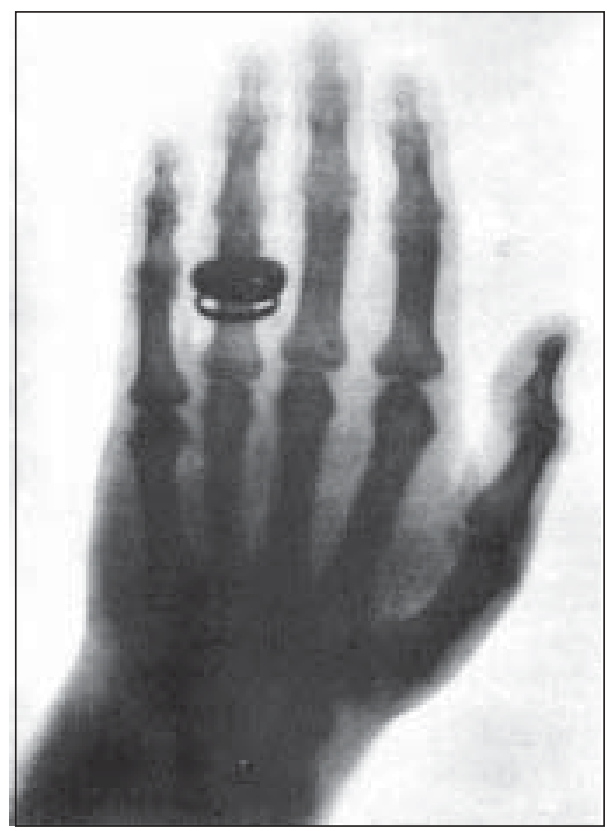

Figura 4. Radiografía de la mano de la esposa de Roentgen.

El 28 de Diciembre manda un informe, ejemplarmente claro, a la Sociedad Físico-médica de Wurzburg, con copias a otros 5 físicos de alto nivel. Uno de ellos, en Viena, mostró el documento a sus ayudantes y uno de estos se lo informó a su padre, editor del diario más importante de Austria. Sorprendentemente, éste se negó a dar el golpe noticioso hasta que tuvo confirmación que la sociedad científica lo había aprobado. La noticia periodística, publicada en 6 de Enero de 1886, fue explosiva y desató un torrente de publicaciones legas y científicas y ensayos en los laboratorios de física, donde los tubos catódicos eran instrumental corriente. Así, al día siguiente se aplicaron los rayos-X para extraer astillas metálicas incrustadas en la mano de un obrero a raíz de una explosión. A fines de Enero tuvo lugar la presentación pública en la Sociedad de Wurzburg donde Kölliker, famoso anatomista, propone el nombre de rayos Roentgen.
Entre Enero y Febrero aparecen traducciones en todo el mundo y a fines de Marzo, se hace en Chile la primera radiografía de Iberoamérica, con la particularidad que los profesores Arturo Salazar y Luis Zegers de la Escuela de Física y Matemáticas improvisaron un tubo de Crookes añadiendo un ánodo externo a una ampolleta de alumbrado.

En pocos meses aparecieron ofertas de equipo comerciales y con ello, iniciativas pintorescas como la venta de ropa interior de mujer opaca a los rayos-X y proyectos de ley para prohibir la incorporación de rayos a los anteojos de ópera.

Roentgen rechazó patentar su descubrimiento, por considerarlo propiedad de la humanidad y en 1901 recibió el primer premio Nobel de Medicina. Es importante destacar que este revolucionario descubrimiento se debió a la observación de un detalle accidental por un hombre curioso y obsesivo sin mayores recursos que los usuales en un laboratorio de física de la época.

Sería ocioso comentar el enorme avance diagnóstico que los rayos Roentgen significaron para la tisiología, pero conviene recordar que la terapéutica siguió siendo limitada por varias décadas, aunque la posibilidad de detectar tuberculosis leves permitió obtener mejores resultados.

En Estados Unidos el enfrentamiento de la tuberculosis tuvo algunas características especiales que conviene conocer. Quien mejor representa estas tendencias es, probablemente, Edward L. Trudeau (Figura 5), médico con un serio historial de tuberculosis y una actividad y perseverancia notables. Su madre y un hermano murieron de tuberculosis y él personalmente tuvo sucesivamente un absceso frío, adenopatías y en 1873 una tuberculosis pulmonar. Como esta mejorara después de una excursión de caza a la montañas Adirondaks, concibió formar un sanatorio en la zona y en 1882 construyó una cabaña con dos camas al lado del lago Saranac. A pesar de un incendio, su obra creció rápidamente con un laboratorio de investigación microbiológica incluido. A diferencia de lo sucedido en Europa, Trudeau logró unir los sanatorios americanos en la Sociedad Sanatorial Americana de orientación científica que, con el tiempo, se transformaría en la Sociedad Trudeau y finalmente en la Sociedad Torácica Americana (ATS) organismo señero de la neumología. También participó en la creación de la Asociación Nacional para el Estudio y Prevención de la Tuberculosis, que luego se transformó en la American Lung Association, orientada a incorporar a la 


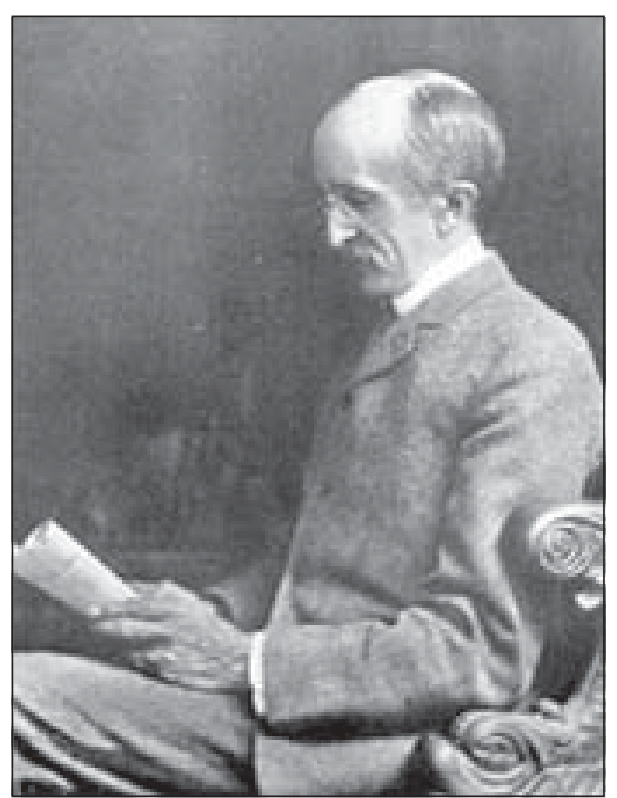

Figura 5. Edward L. Trudeau (1848-1915).

población y a las autoridades en la lucha contra la tuberculosis, lo que se hizo con estrategias empresariales de reclutamiento masivo de voluntarios; presiones políticas al gobierno y parlamento para mejoras habitacionales, nutricionales, condiciones laborales y pasteurización de la leche. Trudeau y sus colaboradores lograron transformar las donaciones a la Sociedad en la caridad de moda e implantaron la costumbre de los sellos de navidad y promovieron un estilo de vida y combate frontal al contagio a través de propaganda constante y agresiva. (Figuras 6 a y b).
A principios de este siglo la cruz de Lorena de las cruzadas, que figura en el logotipo de nuestra sociedad, se adoptó como emblema de la cruzada antituberculosis.

No es posible determinar si fueron algunas de las acciones antes mencionadas $\mathrm{u}$ otras no identificadas las que incidieron en la mortalidad por tuberculosis, pero se ha planteado que la visión social y epidemiológica de la tisiología fueron factores significativos. En los países con mayor desarrollo se observó a lo largo del siglo XIX un leve, pero mantenido, descenso de mortalidad que se hizo más pronunciado hacia fines de ese siglo y primera mitad del siguiente, cuando se produjo el primer quiebre neto e importante con el descubrimiento de antibióticos y quimioterápicos. Paradójicamente, este éxito terminó con la tisiología como especialidad clínica, ya que la eficacia de esta arma dependía de una alta cobertura, para lo cual tenía que involucrar a médicos generales y enfermeras dentro de un programa organizado Debe destacarse que los tisiólogos, sacrificando su campo exclusivo, fueron fuertes impulsores de este cambio.

A principios del siglo XX Albert Calmette y Camille Guérin en Francia decidieron emprender la búsqueda de una vacuna contra la tuberculosis y creyeron haberla alcanzado al obtener una cepa de bacilos atenuados por sucesivos cultivos en bilis. La promesa se cumplió parcialmente, observándose efectos variables en diferentes países, probablemente por diferencias epidemiológicas de las poblaciones estudiadas y la calidad de las cepas empleadas. En 1930 una confusión de cepas en Lubeck, Alemania, provocó la muerte de cerca de un tercio de 251 niños
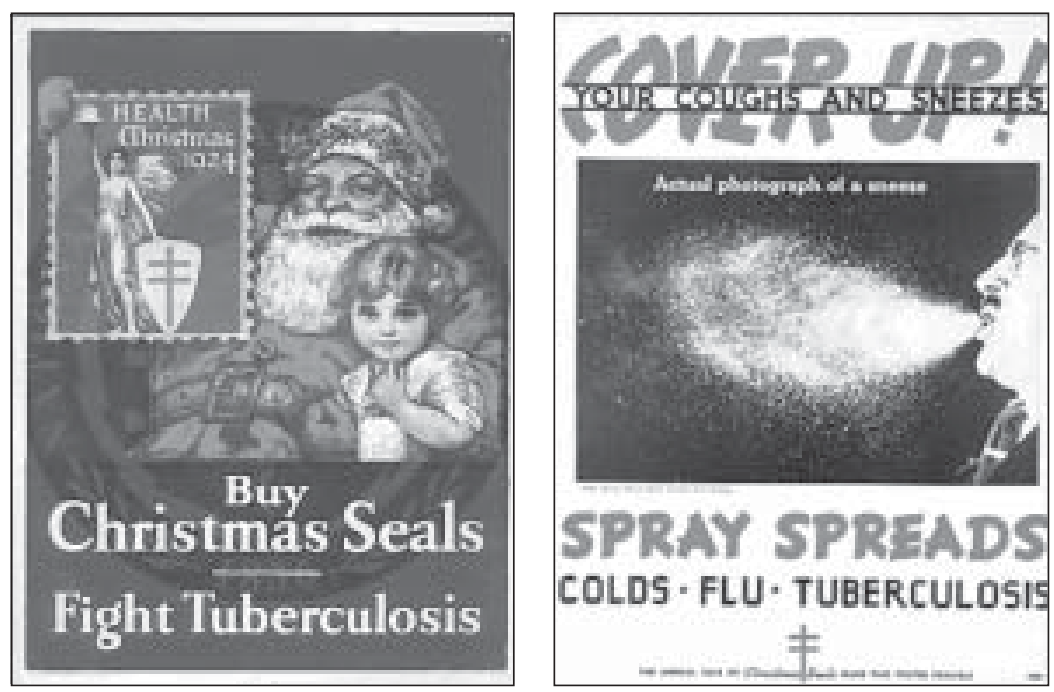

Figuras 6 a y b. Sellos de Navidad, American Thoracic Society. 
vacunados con las consiguientes recriminaciones y desconfianza sobre el método, que en nuestro país ha tenido un efecto positivo en tuberculosis infantil.

Y así llegamos a la década de los 40 en que por primera vez aparece un arma eficaz contra la plaga. No voy a entrar en detalles respecto a los antibióticos, que merecerían una conferencia aparte y cuyo empleo es parte de la experiencia de todos nosotros, pero quiero destacar un par de puntos.

El descubridor de la estreptomicina Selman Waksman era un químico de suelos, enfocado al rol de los microbios en la biología del suelo. Constató la desaparición de patógenos de la tierra por acción de antibióticos producidos por hongos locales, pero como no estaba interesado en enfermedades humanas estuvo en 5 ocasiones, entre 1916 y 1943 ante hallazgos que pudieron haberle orientado a su aplicación clínica. Por su encargo, su ayudante Albert Schatz estudió el efecto de la estreptomicina sobre diversos gérmenes, incluido el bacilo de Koch, pero tampoco le dio proyección clínica, a pesar que ésta fue sugerida por un hijo de Waksman, estudiante de tercer año de medicina, todo lo cual ilustra que la excesiva focalización de la investigación y la falta de contacto con otras disciplinas pueden limitar sus proyecciones. Fue por presión del clínico William Feldman y del microbiólogo Corwin Hinshaw, que Waksman accedió a suministrarles estreptomicina para ensayos, primero en animales y luego en una paciente muy avanzada, que sanó. Es interesante comentar que -posteriormente- el primer estudio clínico aleatorio y controlado realizado en el mundo fue sobre la terapia de la tuberculosis con

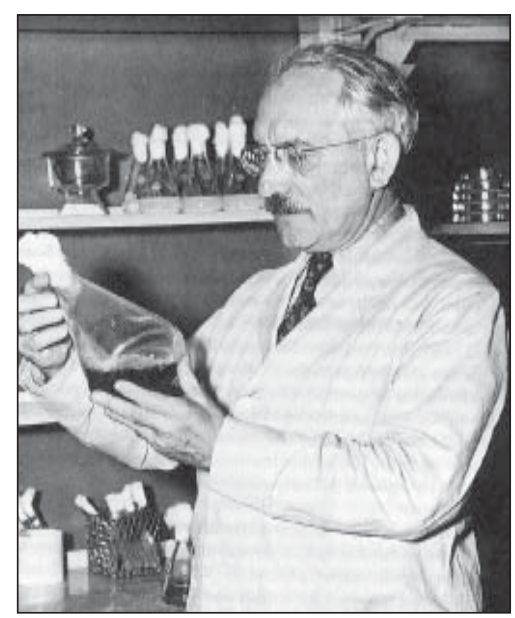

Figura 7. Selman Waksman. estreptomicina en 1948, gracias a la buena preparación epidemiológica de quienes trabajaban alrededor de la tuberculosis.

El otro punto que quiero destacar, por ser poco conocido, es que la primera publicación en el mundo sobre tratamiento multiasociado de la tuberculosis fue de un tisiólogo chileno, Arturo Rodríguez. Por tratarse de una publicación nacional no tuvo impacto mundial, pero Schatz, que en un viaje a Chile tuvo acceso al artículo, lo reconoció públicamente (Figura 8).

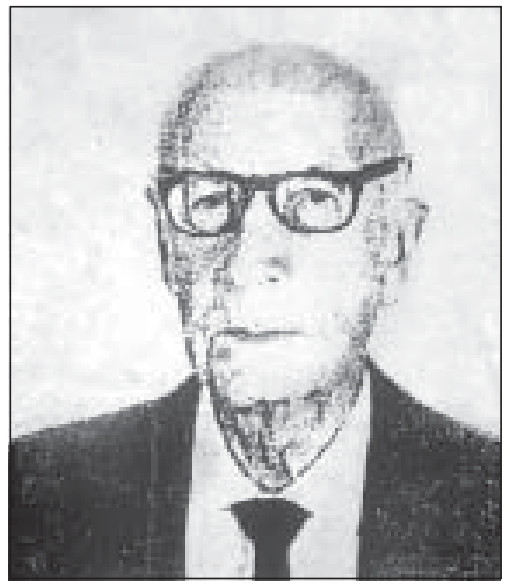

Figura 8. Dr. Arturo Rodríguez.

A estas altura nos encontramos con otra paradoja: si no fuera por el desastre de la segunda guerra mundial, es posible que la tisiología se hubiera extinguido y que la neumología hubiera demorado su aparición o aun no se hubiera desarrollado.

Un arma decisiva en esta conflagración fueron los aviones que volaban a gran altura. El gobierno de Estados Unidos, que creó organismos para investigar en el desarrollo de armamentos, tuvo la perspicacia de dar igual importancia a la investigación de la fisiología respiratoria y circulatoria de quienes tenían que conducir estos aviones. Cabe destacar que hasta ese entonces ningún texto de fisiología dedicaba un capítulo a la función pulmonar y a lo más mencionaban en algún párrafo la espirometría de Hutchinson.

Bajo la presión de la guerra las estructuras existentes fueron obligadas a facilitar sus instalaciones, se aportaron nuevos recursos y se reclutaron múltiples investigadores, no por lo que sabían o estaban haciendo, sino por su capacidad para investigar en lo que no sabían, 
asignándoseles los temas de ciencia aplicada que aparecían como necesarios.

Así, para enfrentar la hipoxia de altura observada en los aviones de combate, que no tenían cabina altimática porque complicaba su construcción y operación, y para determinar los efectos de la aceleración sobre la mecánica tóraco-pulmonar, se reclutó a Herman Rahn, que estaba dedicado a los cambios de color de los reptiles; a Wallace Fenn que estudiaba la tirosinasa en huevos de saltamontes y a Arthur Otis quien trabajaba en potasio en músculos. Estos tres investigadores demostraron que, aunque no conocían el tema que se les encargó, dominaban el método de cómo interrogar a la naturaleza, sentando las bases de la mecánica tóraco-pulmonar y desarrollando las ecuaciones del aire alveolar. Estas últimas fueron consideradas como secreto de guerra ya que permitían calcular la cantidad exacta de balones de oxígeno que el avión debía llevar dejando espacio para las bombas.

André Cournand simplificó y difundió la punción arterial y Glen Millikan, solidarizando con los aviadores inventó el oxímetro de oreja transcutáneo, para evitarles el trauma. Richard Riley caracterizó la relación V/Q y Lilienthal diseñó el medidor de $\mathrm{CO}$ y así podría seguirse con una larga lista, de la cual sólo quiero destacar a una persona: Julius Comroe (Figura 9).

Este investigador fue destinado durante la guerra a resolver problemas con la fabricación de fosgeno y los mecanismos de la disnea, pero su mérito fue mucho más allá de estas tareas específicas. Con una visión y tenacidad extraordinarias luchó con autoridades civiles, militares y científicas para convencerlas que, terminadas

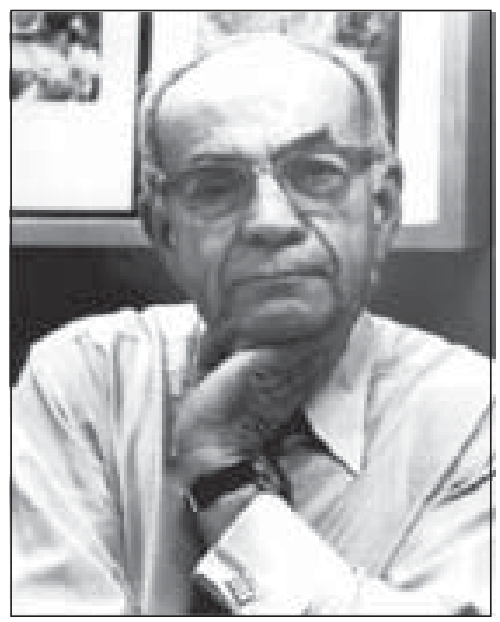

Figura 9. Dr. Julius H. Comroe jr (1911-1984). las hostilidades, todos los avances científicos y técnicos logrados, debían trasmitirse a la sociedad civil y a la medicina. La tarea no fue fácil, pero Comroe no se rindió y terminó por lograr su meta y aun a imponer la investigación respiratoria en un programa permanente de los National Institutes of Health de Estados Unidos. Todo esto unido a una bien fundamentada campaña, para destruir el mito que la investigación aplicada era una actividad inferior a la investigación básica, logró entusiasmar a una pléyade de investigadores jóvenes a hacer ambos tipos de investigación en el área respiratoria. Su libro "The Lung" fue el texto de fisiología y fisiopatología que en las décadas del '50 al '70 sirvió de Biblia a los neumólogos del mundo y sus artículos, agrupados bajo el titulo de "Retrospectroscope", representan las más atrayentes exposiciones de historia de las ideas y hechos de la medicina.

No vamos a enumerar todos los destacados especialistas que así se formaron o se polarizaron a respiratorio ni sus aportes que, sumados a lo esencial de la tisiología, dieron origen a nuestra especialidad. A todos los hemos conocido por sus publicaciones y a muchos como expositores en nuestros congresos o como becarios en sus laboratorios. Como característica común tienen que no han trepidado en hacer investigación básica para contestar preguntas clínicas o responder a las demandas de la cirugía de pulmón y corazón. Sólo recordaremos unos pocos:

- Paul Astrup y John W. Severinghaus, que incorporaron a la clínica el equilibrio ácidobase.

- R. Doll y R. Peto, que nos enseñaron el valor de la epidemiología en la lucha contra el tabaco, la EPOC y el cáncer.

- John A. Clements, que abrió el mundo del surfactante.

- Peter T. Macklem, impulsor del concepto de pequeña vía aérea.

- A.P. Fishman, investigador en la circulación pulmonar.

- Eleanor Baldwin y Dickinson W. Richards, generadores de los primeros valores de referencia modernos para la espirometria.

- Leland C. Clark, inventor del electrodo de $\mathrm{O}_{2}$, base de nuestros actuales equipos de gases. 
- Jere Mead, investigador en mecánica pulmonar.

- John B. West, líder en fisiología de altura.

- Joseph Milic-Emili, autoridad en flujo aéreo.

Todo este caudal fue captado por la tisiología que abrió las puertas de sus sociedades científicas a estas investigaciones y atrajo a esta nueva generación de médicos y conocimientos, para formar una nueva especialidad.

Creo que lo expuesto explica dos aspectos que, aparte de la dedicación a un área de la medicina, caracterizan a nuestra especialidad: el énfasis en dominar las bases fisiopatológicas de los problemas clínicos y la preocupación por los aspectos epidemiológicos y sociales que nos legó la tisiología. Conviene destacar que esta actitud ha sido la base sobre la cual se han podido desarrollar, con una visión poblacional los programas de tratamiento controlado de la tuberculosis a nivel mundial.
Espero haber logrado convencerlos que somos continuadores de generaciones pasadas, que nos han dejado herencias que debemos cuidar y que si hemos alcanzado la altura en que hoy estamos es porque, como dijo Newton, "estamos parados sobre hombros de gigantes”.

\section{Bibliografía}

1.- AMERICAN PHYSIOLOGICAL SOCIETY. Handbook of Physiology. Eds: Fenn WO y Rahn H Section 3: Respiration, Volume 1, Waberly Press, Inc, Baltimore, Maryland, USA. 1964.

2.- COMROE Jr J H. Retrospectroscope. Insights into Medical Discovery. Von Gehr Press, Menlo Park, California 1977.

3.- http://www.thoracic.org/sections/aboutats/centennial/ retrospectroscope/articles/index.html

4.- MENA G. El Tránsito de la Tisiología a la Especialidad Actual en Chile. Rev Chil Enf Respir 1989; 5: 99-108.

5.- BARROS M. Sociedad Chilena de Enfermedades Respiratorias: 75 años de historia. Rev Chil Enf Respir 2005; 21: 5-7.

6.- FARGA V. La Conquista de la Tuberculosis. Rev Chil Enf Respir 2004; 20: 101-8.

Correspondencia a:

Dr. Edgardo Cruz M.

E-mail: ecruz@vtr.net

Depto de Enfermedades Respiratorias. Pontificia Universidad Católica.

Marcoleta $345,4^{\circ}$ piso

Santiago-Chile 